\title{
Ordered states of adatoms on graphene
}

\author{
Vadim V. Cheianov, ${ }^{1}$ Olav Syljuåsen, ${ }^{2}$ B. L. Altshuler,,${ }^{1,3}$ and Vladimir Fal'ko ${ }^{1}$ \\ ${ }^{1}$ Physics Department, Lancaster University, Lancaster LA1 4YB, United Kingdom \\ ${ }^{2}$ Institute for Physics, University of Oslo, P.O. Box 1048 Blindern, N-0316 Oslo, Norway \\ ${ }^{3}$ Physics Department, Columbia University, 538 West 120th Street, New York, New York 10027, USA \\ (Received 16 September 2009; revised manuscript received 19 November 2009; published 30 December 2009)

\begin{abstract}
We show that a dilute ensemble of epoxy-bonded adatoms on graphene has a tendency to form a spatially correlated state accompanied by a gap in graphene's electron spectrum. This effect emerges from the electronmediated interaction between adatoms with a peculiar $1 / r^{3}$ distance dependence. The partial ordering transition
\end{abstract} \\ is described by a random bond three-state Potts model.
}

DOI: 10.1103/PhysRevB.80.233409

PACS number(s): 73.20.Hb, 68.35.Rh, 73.61.-r

Graphene (monolayer of graphite) is a truly twodimensional crystal, just one atom thick. ${ }^{1}$ It is a gapless semiconductor with charge carriers mimicking relativistic dynamics of massless Dirac fermions, ${ }^{2}$ a peculiarity dictated by the bonding of carbon atoms into a highly symmetric honeycomb lattice. Graphene can host various adsorbents, in particular atoms, retaining its own structural integrity. Such chemisorbed atoms (adatoms) may strongly affect electronic properties of graphene ${ }^{3-9}$ introducing symmetry-breaking perturbations into the lattice. The type of symmetry breaking depends on the position of the adatom in the hexagonal unit cell of the crystal. In particular, alkali atoms position themselves over the centers of the hexagons. ${ }^{10}$ Oxygen, nitrogen, boron, or an additional carbon ${ }^{11}$ prefer "epoxy" bonded positions (e-type) and reside above the middle of a carboncarbon bond. Atomic hydrogen and halogens reside in the symmetric on-site position above the carbon (s-type). ${ }^{12}$ It has also been noticed that a pair of hydrogen atoms on the neighboring sites of graphene lattice forms a stable $\mathrm{H}-\mathrm{H}$ dimer which acts as an e-type adsorbent. ${ }^{13}$

Here we predict that an ensemble of e-type adatoms (those perturbing $\mathrm{C}-\mathrm{C}$ bonds) tend to order, mimicking a superlattice structure, even when graphene coverage by adsorbents is low. The underlying mechanism is a long-range electron-mediated interaction between adatoms similar to the Ruderman-Kittel-Kasuya-Yoshida (RKKY) exchange between localized spins in metals. ${ }^{14}$ The effect is peculiar to graphene. Unlike metals, charge neutral graphene has a pointlike Fermi surface positioned in the corners $\mathbf{K}$ and $\mathbf{K}^{\prime}$ $=-\mathbf{K}$ of the hexagonal Brillouin zone-called valleys. The electron density of states vanishes at the Fermi level. As a result, the Friedel oscillations in charge neutral graphene are commensurate with its honeycomb lattice and decay as the inverse cube of the distance to the adatom. ${ }^{15}$ We show that such an interaction in a dilute ensemble of e-type adsorbents may result in their partial ordering associated with a superlattice structure with the unit cell three times larger than in graphene, as illustrated in Fig. 1. We present our results in the following order. Starting with a particular tight-binding model for an e-type adsorbent, we determine the form of a perturbation it creates for the electrons in graphene. Using group theory we classify such interactions beyond a specific microscopic model and determine the conditions under which RKKY interaction between adatoms leads to a partially ordered state with a gapful electronic spectrum. We conclude by discussing experimental signatures of the effect.

The $\pi$-electron band in graphene is well described by the closest-neighbor tight-binding model, $H_{0}$ $=\gamma_{0} \Sigma_{\left[\mathbf{r}_{A} \mathbf{r}_{B}\right]}\left\{c_{\mathbf{r}_{A}}^{\dagger} c_{\mathbf{r}_{B}}+\right.$ H.c. $\}$ with hoping parameter $\gamma_{0} \sim 3 \mathrm{eV} .{ }^{2}$ The sum runs over all pairs $\left[\mathbf{r}_{A} \mathbf{r}_{B}\right]$ of neighboring $A$ and $B$ sites of the lattice and $c^{\dagger} / c$ are the on-site electron creation/ annihilation operators. ${ }^{16}$ An e-type adatom attached to the bond between the sites $\mathbf{r}_{A}^{\prime}$ and $\mathbf{r}_{B}^{\prime}$ creates a local perturbation

$$
H_{a}=\gamma_{0} \xi_{1}\left(c_{\mathbf{r}_{A}^{\prime}}^{\dagger} c_{\mathbf{r}_{A}^{\prime}}+c_{\mathbf{r}_{B}^{\prime}}^{\dagger} c_{\mathbf{r}_{B}^{\prime}}\right)+\gamma_{0} \xi_{2}\left(c_{\mathbf{r}_{A}^{\prime}}^{\dagger} c_{\mathbf{r}_{B}^{\prime}}+c_{\mathbf{r}_{B}^{\prime}}^{\dagger} c_{\mathbf{r}_{A}^{\prime}}\right),
$$

where $\xi_{1}, \xi_{2}<1$ determine how the adatom affects the onsite potential $\left(\xi_{1}\right)$ and the electron hopping amplitude between the sites $\left(\xi_{2}\right)$.

The long-range RKKY interaction between two adatoms is due to the perturbation of the electron spectrum near the Fermi energy and is adequately described in terms of the

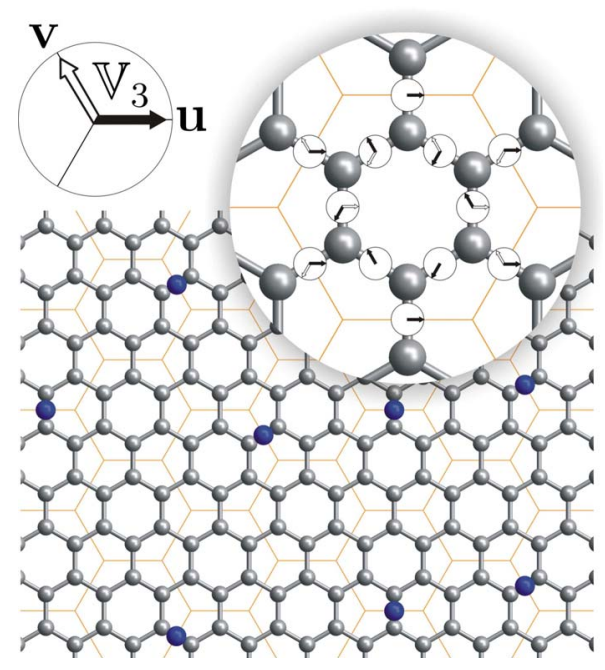

FIG. 1. (Color online) An ordered ensemble of adatoms on graphene lattice: all impurity atoms occupy positions at the intersection of graphene bonds and the bonds of the underlying fictitious superlattice. The inset shows how the superlattice originates from the scattering matrix $\hat{W}$, Eq. (3). To each bond there corresponds a pair of vectors $(\mathbf{u}, \mathbf{v})$ forming a periodic pattern with three times graphene lattice's period. 
TABLE I. Symmetry-based classification of RKKY interactions for different types of adatoms. The parameterization of the adatom scattering matrix $\hat{W}_{a}$ is given in terms of an orbit $\mathcal{W}_{i}$ of the lattice symmetry group in a given irrep $i$. The potential $\Omega_{a b}^{(i)}$, is a contribution of the given orbit to the adatom-adatom interaction, Eq. (5). The last column indicates ordering favored by the given interaction term.

\begin{tabular}{|c|c|c|c|c|}
\hline Irrep $i$ & Orbit $\mathcal{W}_{i}$ & $\Omega_{a b}^{(i)}$ & Type: position & Partial order \\
\hline$A_{1}$ & 1 & $-1 / 2$ & All & \\
\hline$B_{2}$ & $s \Sigma_{z} \Lambda_{z}, \quad s= \pm 1$ & $s_{a} s_{b}$ & s: & Sublattice \\
\hline$E_{1}^{\prime}$ & $\Sigma_{z}(\boldsymbol{\Lambda} \cdot \mathbf{u}), \quad \mathbf{u} \in \mathbb{V}_{3}$ & $\mathbf{u}_{a} \cdot \mathbf{u}_{b}$ & & $3 \times$ unit cell superlattice \\
\hline$E_{2}$ & $\Lambda_{z}(\boldsymbol{\Sigma} \cdot \mathbf{v}), \quad \mathbf{v} \in \mathbb{V}_{3}$ & $\mathbf{v}_{a} \cdot \mathbf{v}_{b}-\frac{3}{2}\left(\mathbf{n} \cdot \mathbf{v}_{a}\right)\left(\mathbf{n} \cdot \mathbf{v}_{b}\right)$ & & None \\
\hline$G$ & $(\mathbf{v} \times \mathbf{\Sigma})(\mathbf{u} \times \boldsymbol{\Lambda}), \quad \mathbf{u}, \mathbf{v} \in \mathbb{V}_{3}$ & $\frac{1}{2}\left(\mathbf{u}_{a} \cdot \mathbf{u}_{b}\right)\left[3\left(\mathbf{n} \cdot \mathbf{v}_{a}\right)\left(\mathbf{n} \cdot \mathbf{v}_{b}\right)-\left(\mathbf{v}_{a} \cdot \mathbf{v}_{b}\right)\right]$ & & None \\
\hline$E_{1}^{\prime \prime}$ & $\begin{array}{c}s \Sigma_{x}(\boldsymbol{\Lambda} \cdot \hat{s} \mathbf{u})+\Sigma_{y}(\boldsymbol{\Lambda} \times \hat{s} \mathbf{u}), \quad \mathbf{u} \in \mathbb{V}_{3}, \\
\hat{s}=\operatorname{diag}(1, s) \quad s= \pm 1\end{array}$ & $\frac{2-s_{a} s_{b}}{2}\left[\left(\mathbf{n} \cdot \mathbf{u}_{a}\right)\left(\mathbf{n} \cdot \mathbf{u}_{b}\right)-s_{a} s_{b}\left(\mathbf{n} \times \mathbf{u}_{a}\right)\left(\mathbf{n} \times \mathbf{u}_{b}\right)\right]$ & & None \\
\hline
\end{tabular}

four-component field, $\psi(\mathbf{r})=\left(\psi_{1}, \psi_{2}, \psi_{3}, \psi_{4}\right)^{T}$, which is smooth on a scale of the lattice constant, $a$

$$
c_{\mathbf{r}}=\left(\frac{3}{4}\right)^{1 / 4} a \times\left\{\begin{array}{lc}
e^{i \mathbf{K r}} \psi_{1}(\mathbf{r})+e^{-i \mathbf{K r}} \psi_{4}(\mathbf{r}), & \mathbf{r}=\mathbf{r}_{A} \\
e^{i \mathbf{K r}} \psi_{2}(\mathbf{r})+e^{-i \mathbf{K r}} \psi_{3}(\mathbf{r}), & \mathbf{r}=\mathbf{r}_{B} .
\end{array}\right.
$$

In the presence of an adatom $\psi(\mathbf{r})$ obeys the Hamiltonian ${ }^{17}$

$$
\begin{gathered}
\hat{H}=v \int \psi^{\dagger}(\boldsymbol{\Sigma} \cdot \hat{\mathbf{p}}) \psi d^{2} r+\hbar v a \psi^{\dagger}\left(\mathbf{r}_{a}\right) \hat{W}_{a} \psi\left(\mathbf{r}_{a}\right), \\
\hat{W}_{a}=\lambda_{A_{1}}+\lambda_{E_{1}^{\prime}} \Sigma_{z}\left(\boldsymbol{\Lambda} \cdot \mathbf{u}_{a}\right)+\lambda_{E_{2}} \Lambda_{z}\left(\boldsymbol{\Sigma} \cdot \mathbf{v}_{a}\right) \\
+\lambda_{G}\left(\boldsymbol{\Lambda} \times \mathbf{u}_{a}\right)\left(\boldsymbol{\Sigma} \times \mathbf{v}_{a}\right)
\end{gathered}
$$

with $v=\sqrt{3} a \gamma_{0} / 2 \hbar$ and $\Sigma_{x, y}=\sigma_{x, y}^{\mathrm{s}} \otimes \sigma_{z}^{\mathrm{v}}$. The Pauli matrices $\sigma_{x}^{\alpha}, \sigma_{y}^{\alpha}$ and $\sigma_{z}^{\alpha}$ operate on the valley $(\alpha=\mathrm{v})$ or sublattice $(\alpha=\mathrm{s})$ indices. Together with $\Sigma_{z}=\sigma_{z}^{\mathrm{s}} \otimes 1^{\mathrm{v}}$, matrices $\Sigma_{x, y}$ form a representation of the $\mathrm{SU}_{2}^{\Sigma}$ algebra. The first term in $H$ determines the Dirac electronic spectrum. It possesses a "flavor" $\mathrm{SU}_{2}^{\Lambda}$ symmetry generated by the three matrices $\Lambda_{x, y}=\sigma_{z}^{\mathrm{s}} \otimes \sigma_{x, y}^{\mathrm{v}}$ and $\Lambda_{z}=\mathbf{1}^{\mathrm{s}} \otimes \sigma_{z}^{\mathrm{v}}$, satisfying $\left[\Lambda_{i}, \Sigma_{j}\right]=0$. This symmetry manifests of the conservation of the electron's valley index. Matrices $\Sigma, \Lambda$, and their products $\Lambda_{i} \Sigma_{j}$ can be arranged into irreducible representations (irreps) of the symmetry group $\mathcal{G}$ of the honeycomb lattice, ${ }^{18,19}$ which includes lattice translations, $C_{6 v}$ rotations and mirror reflections. All operators $\Sigma_{i}$ and $\Lambda_{j}$ change signs upon time inversion, therefore only products $\Lambda_{i} \Sigma_{j}$ are time-inversion symmetric and can appear in the scattering matrix ${ }^{20} \hat{W}_{a}$, Eq. (2), describing static perturbations ${ }^{21,22}$ created by adatoms.

For an adatom of a general symmetry type, $\hat{W}_{a}$ can be expanded into orbits in the irreps ${ }^{23}$ of $\mathcal{G}, \hat{W}_{a}=\Sigma \lambda_{i} \mathcal{W}_{i}$. The classification of orbits by the irrep and the symmetry type of adatom is given in the second column of Table I. In particular, the matrix $\hat{W}_{a}$ of an e-type adatom (or the $\mathrm{H}-\mathrm{H}$ dimer) is expanded into orbits in the irreps $A_{1}, E_{1}^{\prime}, E_{2}$, and $G$, corre- sponding to the four terms in Eq. (3). Vectors $\mathbf{u}$ and $\mathbf{v}$ in Eq. (3) take values in the set $V_{3}$ shown in Fig. 1: three unit vectors on the $x-y$ plane, at $120^{\circ}$ angles. Each given bond of graphene lattice is characterized by a pair $(\mathbf{u}, \mathbf{v})$ as shown in Fig. 1. The distribution of $\mathbf{u}$ and $\mathbf{v}$ forms a periodic pattern with three times the graphene lattice period: the intervalley scattering implies the momentum transfer $\Delta \mathbf{K}=\mathbf{K}-\mathbf{K}^{\prime}$ such that $3 \Delta \mathbf{K}$ is a reciprocal lattice vector. Thus, $\hat{W}_{a}$ in the e-type case is periodic on a superlattice, whose unit cell is three times as big as graphene's and contains nine distinguishable $\mathrm{C}-\mathrm{C}$ bonds. The three nontrivial terms in Eq. (3) resemble the coupling of an electron to the in-plane $\Gamma$ - and K-point phonons. ${ }^{19}$ Indeed, the $E_{1}^{\prime}$ term parameterized by the vector $\mathbf{u}$ resembles the effect of the $\mathrm{K}$-point breathing phonon mode. Below, the ensemble average of $\mathbf{u}$ will play the role of the order parameter. The $G$ term is similar to the fourfold degenerate K-point lattice mode. The $E_{2}$ term resembles the uniaxial strain due to a $\Gamma$-point optical phonon. The parameters $\lambda_{i}$ in $\hat{W}_{a}$ are specific for particular atoms. For the model in Eq. (1), $\lambda_{E_{1}^{\prime}}=\lambda_{E_{2}}=\xi_{2}$ and $\lambda_{A_{1}}=\lambda_{G}=\xi_{1}$.

Unlike e-type adatoms, the perturbation introduced by an s-type adatom (e.g., $\mathrm{H}$ on a lattice site) cannot be related to the in-plane phonons, since it explicitly distinguishes A and B sublattices of graphene. The two orbits encountered in the matrix $\hat{W}_{a}$ in this case can be related to the out-of-plane phonons in the presence of a transverse electric field $\left(z \rightarrow-z\right.$ asymmetry): $B_{2}$ resembling the $\Gamma$-point and $E_{1}^{\prime \prime}$ the $\mathrm{K}$-point phonon. In both cases, the $A / B$ residency of an adatom is accounted for by $s= \pm 1$.

Each type of the adsorbents listed in Table I creates Friedel oscillations of the electron density breaking the symmetry in the same way as the adatom does. The polarization caused by one adatom extends over long distances, thus leading to interaction between the adatoms. We investigate such an interaction assuming vanishing carrier density, which at low adatom coverage can be achieved by applying a back- 


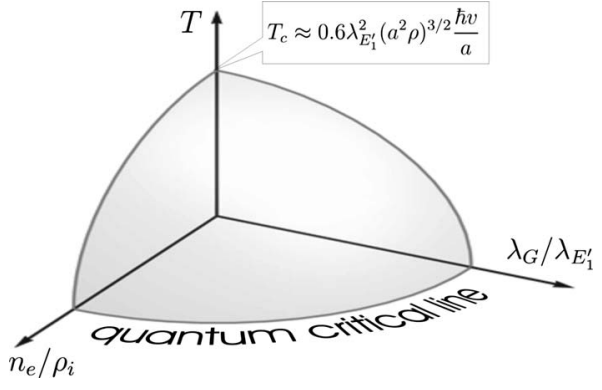

FIG. 2. The schematic phase diagram of the epoxy-bonded adatom system on graphene. The critical surface separates the gapped ordered phase (Fig. 1) from the disordered phase.

gate voltage to the sample. The effect of finite carrier density is discussed qualitatively in the context of the phase diagram shown in Fig. 2. The interaction energy between a pair of adatoms at a distance $\left|\mathbf{r}_{a b}\right| \gg a$ can be expressed through the imaginary time (Matsubara) Green's function of electrons in a clean graphene, $G(\mathbf{r}, \tau)($ Ref. 24$)$ as

$$
\begin{gathered}
F_{a b}=2 \hbar v^{2} a^{2} \operatorname{Tr} \int_{-\infty}^{\infty} d \tau \hat{W}_{a} \hat{G}\left(\mathbf{r}_{a b}, \tau\right) \hat{W}_{b} \hat{G}\left(-\mathbf{r}_{a b},-\tau\right), \\
\hat{G}(\mathbf{r}, \tau)=-\frac{1}{4 \pi} \frac{v \tau+i \boldsymbol{\Sigma} \cdot \mathbf{r}}{\left(v^{2} \tau^{2}+r^{2}\right)^{3 / 2}} .
\end{gathered}
$$

Here, trace is taken over the valley and sublattice indices, and spin degeneracy is taken into account. Equation (4) yields a long-range pair-correlation energy,

$$
\begin{gathered}
F_{a b}=-\frac{\hbar v a^{2}}{4 \pi} \frac{\Omega_{a b}}{r_{a b}^{3}}, \quad \mathbf{n}=\frac{\mathbf{r}_{a b}}{r_{a b}}, \\
\Omega_{a b}=\frac{1}{16} \operatorname{Tr}\left[\hat{W}_{b} \hat{W}_{a}-3 \hat{W}_{a}(\boldsymbol{\Sigma} \cdot \mathbf{n}) \hat{W}_{b}(\boldsymbol{\Sigma} \cdot \mathbf{n})\right] .
\end{gathered}
$$

Interaction between adatoms of the same type depends on both the type of the adatoms and the parameters $\lambda_{i}$ characterizing the adatom-electron coupling in each symmetrybreaking interaction channel $\mathcal{W}_{i}$

$$
\Omega_{a b}=\sum_{i} \lambda_{i}^{2} \Omega_{a b}^{(i)}
$$

For the model in Eq. (3) with $\lambda_{E_{1}^{\prime}}$ larger than other coupling parameters, $\Omega_{a b} \approx \lambda_{E_{1}^{\prime}}^{2} \mathbf{u}_{a} \cdot \mathbf{u}_{b}$, so that the interaction between lateral degrees of freedom of the two adsorbents looks like an isotropic ferromagnetic exchange. Therefore the adatoms tend to occupy preferably bonds with the same $\mathbf{u}$ and form a partially ordered state shown in Fig. 1. The transition to such a state is described by a special case of random exchange three-state Potts model: ${ }^{25}$ "spins" u reside on randomly distributed with density $\rho$ sites and experience pair-wise exchange interaction $-J \mathbf{u}_{a} \cdot \mathbf{u}_{b} / r^{3}$. According to recent cluster Monte-Carlo ${ }^{26}$ studies this model undergoes an orderdisorder transition at a critical temperature $T_{c} \approx 8 \rho^{3 / 2} \mathrm{~J}$. For the Eq. (1) model $T_{c}$ is evaluated as

$$
T_{c} \approx 0.6 \lambda_{E_{1}^{\prime}}^{2}\left(a^{2} \rho\right)^{3 / 2} \frac{\hbar v}{a} .
$$

Note that our theory has nothing to say about the kinetic aspect of the transition. The latter should be sensitive to the ratio $U_{m} / T_{c}$ of the adatom migration barrier $U_{m}$ (Ref. 9) and the critical temperature $T_{c}$. Only for $U_{m} /\left(k_{B} T_{c}\right) \leq 1$ will the predicted transition be fast enough to be experimentally observable.

Consider now effects of other terms in Eq. (6) allowed by the e-type symmetry. These terms are listed in the rows $A_{1}$, $E_{2}$, and $G$ in Table I. The symmetric perturbation parameterized by $\lambda_{A_{1}}$ leads to the repulsion between adatoms regardless of which bonds of the extended supercell they occupy. The coupling parameterized by $\lambda_{E_{2}}$ causes an anisotropic "antiferromagnetic" interaction of the alternative set of Potts "spins" $\mathbf{v}_{a}$. Frustration precludes ordering of $\mathbf{v}_{a}$, which could lead to a unilateral deformation of the lattice. However, a presence of $\lambda_{E_{2}}^{2} \Omega_{a b}^{\left(E_{2}\right)}$ in $\Omega_{a b}$, Eq. (6) does not affect the ordering of vectors $\mathbf{u}_{a}$, at least up to the quadratic order in $\lambda_{E_{2}}$. The isotropic interaction leading to the ordering only competes with the anisotropic interaction between adatoms caused by the last term in $\hat{W}_{a}$, Eq. (3) (the fifth row of Table I). If the latter is strong, $\lambda_{G}>\lambda_{E_{1}^{\prime}}$, it suppresses ordering by frustration. As a result, $T_{c}$ of the order-disorder transition decreases with increasing ratio $\lambda_{G} / \lambda_{E_{1}^{\prime}}$ until it vanishes at a quantum critical point.

Increasing the density $n_{e}$ of mobile carriers in graphene should also suppress $T_{c}$. Indeed, at finite $n_{e}$ the RKKY interaction develops Friedel oscillations, ${ }^{22}$ $F \propto\left(\mathbf{u}_{a} \cdot \mathbf{u}_{b}\right) \sin ^{2}\left(k_{F} r\right) / r^{2}$, which lead to a random sign of the exchange coupling between adatoms at distance $r_{a b}>\sqrt{1 / n_{e}}$. At sufficiently large ratio $n_{e} / \rho$ this effect should completely destroy ordering of Potts spins. These considerations are illustrated by the phase diagram in Fig. 2, where the "quantum critical line" corresponds to the parametric condition $T_{c}\left(n_{e} / \rho, \lambda_{G} / \lambda_{E^{\prime}}\right) \rightarrow 0$. Further analysis of these transitions is beyond the scope of this paper.

The interaction of s-type adsorbents residing on the honeycomb lattice sites (e.g., hydrogen) is described in rows 2 and 6 of Table I. Each s-type adatom can be characterized by the Ising spin $s_{a}$ taking the value +1 or -1 depending on which sublattice A or B it occupies. These spins may, potentially, establish sublattice ordering. However, there will be no ordering of the spins $\mathbf{u}_{a}$ : the interaction described in Table I is anisotropic and causes frustrations. In contrast, a pair of hydrogen atoms forming an $\mathrm{H}-\mathrm{H}$ dimer on the nearest $A / B$ sites $^{13}$ falls into the same symmetry class as e-type adatoms and can establish the same type of ordering. It has been noticed that in hydrogenated graphite both configurations of $\mathrm{H}$ atoms are present. ${ }^{13}$ Since the decomposition of the interaction in Eq. (5) suggests the absence of mutual correlations between adsorbents of the e-type and s-type, we expect ordering of the $\mathrm{H}-\mathrm{H}$ dimers on graphene, even if only a fraction of hydrogens covering the flake is dimerized.

The predicted ordering will strongly influence transport and optical properties of the material. As temperature approaches $T_{c}$ from above large clusters of ordered phase will 
act as intervalley-scattering Bragg mirrors for electrons. Backscattering from such mirrors should lead to a power-law increase in resistivity near $T_{c} \cdot{ }^{27}$ At $T<T_{c}$, the spectrum of electrons becomes gapful,

$$
\varepsilon= \pm \sqrt{v^{2} p^{2}+\Delta^{2}}, \quad \Delta=\frac{\hbar v}{a} \lambda_{E_{1}^{\prime}} \rho a^{2} \gg T_{c}
$$

as can be seen from the mean-field Hamiltonian, ${ }^{28}$ $\langle\hat{H}\rangle=v \Sigma \cdot \mathbf{p}+\hbar v \rho a \lambda_{E_{1}^{\prime}} \Sigma_{z} \boldsymbol{\Lambda} \cdot \mathbf{u}$, valid for $p \ll \hbar \rho^{1 / 2}$. At low carrier density this will lead to the activated transport regime typical of semiconductors.

Partial ordering of e-type adsorbents should also be manifest in the structure of the D-peak in the Raman spectrum. The D-peak is associated with the excitation of one optical phonon at momentum $\mathbf{K}$ (or $\mathbf{K}^{\prime}$ ) and is forbidden by momentum conservation in pristine graphene. In the presence of random scatterers, the D-peak is seen as a low-intensity, $I\left(T>T_{c}\right) \propto \rho \lambda_{E_{1}^{\prime}}^{2}$, feature strongly broadened due to the disorder-induced uncertainty in the emitted phonon momentum. Domains of ordered adsorbent, with the size $L$, will scatter electrons between valleys $K$ and $K^{\prime}$ coherently. This will enhance the intensity of the otherwise forbidden transition, $I\left(T<T_{c}\right) \propto \rho \lambda_{E_{1}^{\prime}}^{2} \times \rho L^{2} \gg I\left(T>T_{c}\right)$, and restrict the uncertainty of the emitted phonon momentum to $\delta q \sim 1 / L$. Thus, one can predict that the ordering of adatoms abruptly enhances and narrows the D-peak in the Raman spectrum. To mention, an observation of a hopping conductivity accompanied by a sharp high-intensity D-line in the Raman spectrum in graphene exposed for a long time to hydrogen atmosphere has been reported in Ref. 8. We predict a similar behavior of the angle-resolved photoemission spectroscopy spectrum of graphene: ordering should strongly enhance the photoemission of electrons from the center of the Brillouin zone at energies close to the Fermi energy.

The work was supported by the Lancaster-EPSRC Portfolio Partnership, EPSRC under Grant No. EP/G041954, and U.S. DOE under Contract No. DE-AC02-06CH11357.
${ }^{1}$ K. S. Novoselov, A. K. Geim, S. V. Morozov, D. Jiang, Y. Zhang, S. V. Dubonos, I. V. Grigorieva, and A. A. Firsov, Science 306, 666 (2004).

${ }^{2}$ A. H. Castro Neto, F. Guinea, N. M. R. Peres, K. S. Novoselov, and A. K. Geim, Rev. Mod. Phys. 81, 109 (2009).

${ }^{3}$ S. Gilje, Song Han, Minsheng Wang, Kang L. Wang, and Richard B. Kaner, Nano Lett. 7, 3394 (2007).

${ }^{4}$ F. Schedin, A. K. Geim, S. V. Morozov, E. W. Hill, P. Blake, M. I. Katsnelson, and K. S. Novoselov, Nature Mater. 6, 652 (2007).

${ }^{5}$ J. O. Sofo, A. S. Chaudhari, and G. D. Barber, Phys. Rev. B 75, 153401 (2007).

${ }^{6}$ J. H. Chen, C. Jang, S. Adam, M. S. Fuhrer, E. D. Williams, and M. Ishigami, Nat. Phys. 4, 377 (2008).

${ }^{7}$ S. Y. Zhou, D. A. Siegel, A. V. Fedorov, and A. Lanzara, Phys. Rev. Lett. 101, 086402 (2008).

${ }^{8}$ D. C. Elias, R. R. Nair, T. M. G. Mohiuddin, S. V. Morozov, P. Blake, M. P. Halsall, A. C. Ferrari, D. W. Boukhvalov, M. I. Katsnelson, A. K. Geim, and K. S. Novoselov, Science 323, 610 (2009).

${ }^{9}$ T. O. Wehling, M. I. Katsnelson, and A. I. Lichtenstein, Phys. Rev. B 80, 085428 (2009), and references therein.

${ }^{10}$ D. Lamoen and B. N. J. Persson, J. Chem. Phys. 108, 3332 (1998).

${ }^{11}$ U. Bangert, M. H. Gass, A. L. Bleloch, R. R. Nair, and A. K. Geim, Phys. Status Solidi A 206, 1117 (2009); J. C. Meyer, C. O. Girit, M. F. Crommie, and A. Zettl, Nature (London) 454, 319 (2008); K. Nordlund, J. Keinonen, and T. Mattila, Phys. Rev. Lett. 77, 699 (1996).

${ }^{12}$ L. Jeloaica and V. Sidis, Chem. Phys. Lett. 300, 157 (1999).

${ }^{13}$ L. Hornekær, E. Rauls, W. Xu, Z. Sljivancanin, R. Otero, I. Stensgaard, E. Laegsgaard, B. Hammer, and F. Besenbacher, Phys. Rev. Lett. 97, 186102 (2006).

${ }^{14}$ M. A. Ruderman and C. Kittel, Phys. Rev. 96, 99 (1954);
T. Kasuya, Prog. Theor. Phys. 16, 45 (1956); K. Yosida, Phys. Rev. 106, 893 (1957).

${ }^{15}$ L. Brey, H. A. Fertig, and S. Das Sarma, Phys. Rev. Lett. 99, 116802 (2007).

${ }^{16}$ We omit spin indices throughout the paper.

${ }^{17}$ E. McCann and V. I. Fal'ko, Phys. Rev. B 71, 085415 (2005).

${ }^{18}$ K. Kechedzhi, E. McCann, V. I. Fal'ko, H. Suzuura, T. Ando, and B. L. Altshuler, Eur. Phys. J. Spec. Top. 148, 39 (2007).

${ }^{19}$ D. M. Basko, Phys. Rev. B 78, 125418 (2008).

${ }^{20}$ Strictly speaking, the second term in the Hamiltonian, Eq. (3), is not well defined due to ultraviolet problems and is written in this form for illustrative purposes. In a more rigorous approach $\hat{W}_{a}$ should be interpreted as a scattering $T$-matrix defining the longdistance asymptotic form of the electron wave scattered off the defect.

${ }^{21}$ E. McCann, K. Kechedzhi, V. I. Falko, H. Suzuura, T. Ando, and B. L. Altshuler, Phys. Rev. Lett. 97, 146805 (2006).

${ }^{22}$ V. V. Cheianov and V. I. Fal'ko, Phys. Rev. Lett. 97, 226801 (2006).

${ }^{23}$ The matrix $\hat{W}_{a}$ transforms as a tensor under the symmetry group of the lattice $\mathcal{G}$. It can be decomposed into projections onto irreducible spaces, $\hat{W}_{a}=\Sigma_{i} \mathcal{W}_{i}$. For an adatom preserving some subgroup of $\mathcal{G}$ each $\mathcal{W}_{i}$ is an invariant under this subgroup, sweeping an orbit of $\mathcal{G}$ in the corresponding irreducible representation as the atom jumps between equilibrium positions.

${ }^{24}$ In the $(k, \omega)$ domain $\hat{G}\left(k, i \omega_{n}\right)=\left[i \omega_{n}-v \mathbf{\Sigma} \cdot \mathbf{k}\right]^{-1}$.

${ }^{25}$ F. Y. Wu, Rev. Mod. Phys. 54, 235 (1982).

${ }^{26}$ V. V. Cheianov, V. I. Fal'ko, O. Syljuåsen, and B. L. Altshuler, Solid State Commun. 149, 1499 (2009).

${ }^{27}$ B. Altshuler, V. Cheainov, and V. Falko (unpublished).

${ }^{28}$ C. Chamon, C. Y. Hou, R. Jackiw, C. Mudry, S. Y. Pi, and A. P. Schnyder, Phys. Rev. Lett. 100, 110405 (2008). 